\title{
Evaluation of the relationship between the level of addiction and exhaled carbon monoxide levels with QT dispersion in smokers
}

\author{
Gamze Keskin', Sibel Tunç Karaman', Okcan Basat ${ }^{1}$
}

\begin{abstract}
INTRODUCTION Smoking increases the risk of arrhythmia. QT dispersion (QTd) is an important indicator for the determination of ventricular arrhythmia. In this study, we aimed to determine the arrhythmia risk by evaluating QTd in smokers and to assess the relationship between the level of nicotine addiction and carbon monoxide (CO) level in the expiratory air.

METHODS This study was designed as a single-center, cross-sectional study. Among the chronic smokers referred to the Smoking Cessation Clinic of a tertiary hospital between October 2019 and January 2020, all those who had no risk factors for cardiac arrhythmias, except smoking, were included in the study. Sociodemographic data and smoking characteristics of the participants were collected and exhaled CO levels were measured. QT intervals were measured in all leads by using a 12-lead standard electrocardiogram (ECG), and heart rate corrected QT (QTc) intervals, QT dispersion (QTd), and corrected QT dispersion (QTcd) were calculated.

RESULTS The mean age of the 250 patients was $37.2 \pm 9.3$ years and the majority of patients $(65 \%)$ were male. The mean amount of smoking was $25.74 \pm 16.03$ packs/year and the mean value of CO was $12.36 \pm 7.06 \mathrm{ppm}$. The mean QTd was $23.83 \pm 13.12 \mathrm{~ms}$, and the mean QTcd was $26.63 \pm 15.02 \mathrm{~ms}$. A statistically significant relationship was found between QTd and QTcd and level of addiction, consumption of sticks/day and packs/year, and exhaled CO values (all $\mathrm{p}<0.001$ ). CONCLUSIONS It was found that as the level of addiction, cigarette use amount, exhaled CO levels, and BMI increased in smokers, QT dispersion and arrhythmia risk increased.
\end{abstract}

AFFILIATION
1 Gaziosmanpașa Training
and Research Hospital,
Department of Family
Medicine, University of Health
Sciences, Istanbul, Turkey
CORRESPONDENCE TO
Sibel Tunç Karaman.
Gaziosmanpașa Training
and Research Hospital,
Department of Family
Medicine, University of Health
Sciences, Mevlana Mahallesi,
885 Sk., Gaziosmanpașa,
Istanbul 34255, Turkey. E-mail:
drsibeltunc@ @otmail.com
ORCID ID: https://orcid.
org/0000-0003-1833-8758
KEYWORDS
arrhythmia, exhaled carbon
monoxide, smoking, QT
dispersion

Received: 21 September 2020 Revised: 1 February 2021

Accepted: 3 February 2021

\section{INTRODUCTION}

Smoking causes severe diseases and death and is one of the most crucial risk factors, particularly for cardiovascular system diseases ${ }^{1}$. A meta-analysis found a threefold increase in the relative risk of sudden cardiac death among current smokers ${ }^{2}$.

There are thousands of substances in cigarette smoke that are pharmacologically active, antigenic, carcinogenic, and addictive, including nicotine and carbon monoxide $(\mathrm{CO})^{3}$. While $\mathrm{CO}$ poses a risk for cardiovascular system diseases by worsening hypoxia, another critical component of a cigarette, nicotine, increases the release of catecholamine, activates the sympathetic nervous system and closes the potassium channels located in the ventricular myocardium, and prolongs and delays repolarization ${ }^{4,5}$.

QT dispersion (QTd) (the difference between the maximum and minimum QT interval) is an easily measured electrocardiographic marker and indicates ventricular electrical instability and variable myocardial repolarization ${ }^{6-8}$. Thus, it is considered an important indicator of the severe risk of ventricular 
arrhythmia ${ }^{6}$. Values $40-50 \mathrm{~ms}$ are accepted as normal and values $>65 \mathrm{~ms}$ are associated with an increased risk of severe ventricular arrhythmia ${ }^{9,10}$. In addition to the increase in a wide variety of cardiovascular diseases, QTd may also increase in non-cardiovascular diseases such as diabetes mellitus, rheumatological diseases, and vitamin D deficiency ${ }^{11-13}$.

Previous studies have investigated the effects of $\mathrm{CO}$ intoxication, smoking-related $\mathrm{CO}$ exposure, and acute and chronic smoking on QT dispersion. It has been shown that these factors may be associated with ventricular arrhythmia ${ }^{14-16}$. However, how QT dispersion is affected by the amount of smoking and addiction level has not been shown.

In this study, we aimed to determine the relationship between the amount of smoking, the level of nicotine dependence, and the exhaled CO level, an indicator of cigarette exposure, with QT dispersion, thus determining the risk of ventricular arrhythmia in smokers.

\section{METHODS}

This study was designed as a single-center, cross-sectional study. Ethical permission to conduct this study was obtained from the Local Ethics Committee. (Approval No:146; 2 October 2019). The study was conducted under the principles of the Declaration of Helsinki. Written informed consent was obtained from all participants.

\section{Study population}

All participants were selected from current chronic smokers who were referred for the first time to the Smoking Cessation Clinic of a tertiary hospital from October 2019 to January 2020. Two hundred and fifty people who had no risk factors for cardiac arrhythmia, except smoking, and agreed to participate, were included in the study. Those aged $<18$ years and those $>65$ years that had a history of known cardiovascular disease, diabetes mellitus, chronic pulmonary disease, and those under medication use (such as antibiotics, antihistamines, antiarrhythmic) that may affect QT distance, were excluded from the study. According to the G-power analysis made in line with the reference studies, the minimum number of participants required for the study was 146 with a $95 \%$ CI.

\section{Data collection tools}

Patient information form

A patient information form was formulated, which included the participants' sociodemographic characteristics (age, gender, marital status, educational level), smoking amounts (sticks/day and packs/year), medical history, BMI (Body Mass Index, $\mathrm{kg} / \mathrm{m}^{2}$ ), and exhaled CO measurement.

\section{Fagerström Test for Nicotine Dependence (FTND)}

The Fagerström test for nicotine dependence (FTND $)^{17}$ and the Turkish validity and reliability study conducted by Uysal et al. ${ }^{18}$ were used to measure the degree of nicotine dependence of the participants. The dependence level was categorized according to the following FTND scores: 0-2 very low; 3-4 low; 5 moderate; 6-7 high; and 8-10 very high.

\section{Exhaled CO measurement}

Exhaled CO is increased in smokers and is a biomarker frequently used in the diagnosis, treatment, and follow-up stages of cigarette dependence ${ }^{19}$. Exhaled $\mathrm{CO}$ measurements (in parts per million, ppm) were performed by authorized healthcare staff using piCO + Smokerlyzer (Bedford Scientific, Maidstone, UK, 2016) devices.

Electrocardiographic examination and evaluated parameters

The 12-lead ECG was performed with the Nihon Kohden brand ECG-1350K model device, at a speed of $25 \mathrm{~mm} / \mathrm{s}$ and $10 \mathrm{~mm} / \mathrm{mV}$, while the participants were at rest in the supine position. All the patients had sinus rhythm. Heart rate and QT intervals of the patients were measured manually by a single investigator on ECG with a ruler and magnifying glass, and no computer program was used. The QT interval between the start of the QRS complex and the end of the $\mathrm{T}$ wave was measured in milliseconds. QT intervals in all leads were evaluated by using 12 lead electrocardiograms. Heart rate corrected QT (QTc) intervals, QTc minimum, and QTc maximum values were then calculated by using Bazett's formula $[\mathrm{QTc}=\mathrm{QT} / \sqrt{ }(\mathrm{RR} / 1 \mathrm{~s})]^{20}$. QTd was defined by calculating the difference between the longest and shortest QT intervals. The corrected QT dispersion (QTcd) was defined by calculating the difference between the longest and shortest QTc intervals.

\section{Statistical analysis}

The IBM SPSS Statistics 22 program was used for 
statistical analysis. The compliance of the parameters to normal distribution was evaluated with the ShapiroWilk test. In addition to descriptive statistical methods (mean, standard deviation, frequency) in more than two group comparisons, one-way ANOVA was used when numerical variables showed normal distribution, and the Kruskal-Wallis test was used when there was no normal distribution. Mann-Whitney U test was used to determine the group that caused the difference. Mann-Whitney U test evaluated the comparisons of normally distributed parameters between two groups. Pearson's correlation analysis was performed to examine the relationships between parameters that conform to a normal distribution, and Spearman's rho correlation analysis was used to examine relationships between parameters that did not conform to a normal distribution. Significance was considered at the level of $p<0.05$.

\section{RESULTS}

The mean age of the 250 participants included in the study was $37.2 \pm 9.3$ years, and $62 \%$ were male $(n=155)$. The mean amount of smoking was $25.74 \pm 16.03$ packs/year and the mean value of exhaled $\mathrm{CO}$ was $12.36 \pm 7.06 \mathrm{ppm}$. The mean BMI was $25.81 \pm 4.15$ $\mathrm{kg} / \mathrm{m}^{2}$. Table 1 presents the sociodemographic and smoking characteristics of the participants.

The mean QTd was $23.83 \pm 13.12 \mathrm{~ms}$, and the mean QTcd was $26.63 \pm 15.02 \mathrm{~ms}$. The electrocardiographic findings of the participants are summarized in Table 2.

Exhaled CO values of male smokers were significantly higher $(p=0.005)$. It was found that as the number of daily cigarettes increased, exhaled CO values increased $(\mathrm{p}<0.001)$.

Table 3 presents differences in QTd and QTed according to the smoking and sociodemographic characteristics. The QTd and QTed values of female smokers were significantly lower than those of the male smokers $(p=0.009 ; p=0.013$, respectively $)$. A significant difference was found regarding the number of cigarettes smoked daily and QTd and QTcd values $(\mathrm{p}<0.001 ; \mathrm{p}<0.001$, respectively). The QTd and QTcd values of those who smoke $\geq 31$ cigarettes per day were significantly higher than those who smoked less $(\mathrm{p}<0.001 ; \mathrm{p}<0.001$, respectively).

As the scores from FTND and amount of cigarette consumption of the participants in terms of sticks/ day and packs/year increased, QTd and QTcd also increased significantly (all $\mathrm{p}<0.001)$. We also observed that as BMI values increased, QTd and QTcd increased significantly $(\mathrm{p}=0.010$ and $\mathrm{p}=0.010$, respectively). A positive and significant relationship was present between QTd and QTcd with exhaled $\mathrm{CO}$ values ( $\mathrm{p}<0.001 ; \mathrm{p}<0.001$, respectively) (Table 3 ).

Table 1. Sociodemographic and smoking characteristics of the study population $(\mathrm{N}=250)$

\begin{tabular}{lccc}
\hline Characteristics & Range & Mean \pm SD \\
\hline Age (years) & $18-65$ & $37.2 \pm 9.3$ \\
BMI (kg/m²) & $16.53-41.77$ & $25.81 \pm 4.15$ \\
Smoking amount (packs/year) & $2-105$ & $25.74 \pm 16.03$ \\
Smoking rate (sticks/day) & $5-90$ & $27.34 \pm 11.5$ \\
FTND score & $0-10$ & $6.64 \pm 2.22$ \\
Exhaled CO level (ppm) & $0-32$ & $12.36 \pm 7.06$ \\
Characteristics & Categories & $n(\%)$ \\
Gender & Female & $95(38.0)$ \\
& Male & $155(62.0)$ \\
Alcohol use & No & $183(73.2)$ \\
Smoking rate (sticks/day) & Yes & $67(26.8)$ \\
& $\leq 10$ & $15(6.0)$ \\
& $11-20$ & $90(36.0)$ \\
Level of dependence & $21-30$ & $82(32.8)$ \\
& $\geq 31$ & $63(25.2)$ \\
& Very low & $13(5.2)$ \\
& Low & $30(12.0)$ \\
& Moderate & $25(10.0)$ \\
& High & $79(31.6)$ \\
& Very high & $103(41.2)$ \\
& & \\
& &
\end{tabular}

BMI: body mass index $\left(\mathrm{kg} / \mathrm{m}^{2}\right)$. FTND: Fagerström test for nicotine dependence. CO: carbon monoxide.

Table 2. Electrocardiographic findings of the study population $(\mathrm{N}=250)$

\begin{tabular}{lc|r} 
Measurements & Range & \multicolumn{1}{c}{ Mean \pm SD } \\
\hline HR (beats/min) & $52-112$ & $74.57 \pm 10.66$ \\
OT max (ms) & $300-440$ & $367.19 \pm 26.26$ \\
OT min (ms) & $275-420$ & $343.36 \pm 26.93$ \\
OTd (ms) & $4-64$ & $23.83 \pm 13.12$ \\
OTc max (ms) & $293-503$ & $406.67 \pm 26.98$ \\
OTc min (ms) & $279-467$ & $380.04 \pm 26.51$ \\
OTcd (ms) & $4-85$ & $26.63 \pm 15.02$
\end{tabular}

HR: heart rate. OT max: QT maximum. OTc max: corrected QT maximum. OT min: QT minimum. QTc min: corrected QT minimum. QTd: QT dispersion. QTcd: corrected QT dispersion. 
Table 3. Relationship between QTd and QTed and smoking and sociodemographic characteristics $(\mathrm{N}=\mathbf{2 5 0})$

\begin{tabular}{|c|c|c|c|}
\hline Characteristics & & QTd & QTcd \\
\hline \multirow[t]{2}{*}{ Age } & Spearman rho & 0.111 & 0.093 \\
\hline & $\mathrm{p}$ & 0.081 & 0.142 \\
\hline \multirow[t]{2}{*}{ BMI } & Spearman rho & 0.162 & 0.163 \\
\hline & $\mathrm{p}$ & $0.010^{*}$ & $0.010^{*}$ \\
\hline \multirow[t]{3}{*}{ Gender } & Female & $21.22(12.12)$ & 23.67 (13.6) \\
\hline & Male & $25.43(13.48)$ & 28.44 (15.6) \\
\hline & $\mathrm{p}^{\mathrm{a}}$ & $0.009^{*}$ & $0.013^{*}$ \\
\hline \multirow{2}{*}{$\begin{array}{l}\text { Total amount } \\
\text { of smoking } \\
\text { (pack/years) }\end{array}$} & Spearman rhoc & 0.328 & 0.312 \\
\hline & $\mathrm{p}$ & $<0.001^{*}$ & $<0.001^{*}$ \\
\hline \multirow{6}{*}{$\begin{array}{l}\text { Cigarettes per } \\
\text { day }\end{array}$} & $\leq 10$ & $10.27 \pm 4.38$ & $11.67 \pm 4.81$ \\
\hline & $11-20$ & $20.11 \pm 11.54$ & $22.44 \pm 13.4$ \\
\hline & $21-30$ & $26.34 \pm 12.41$ & $29.73 \pm 14.7$ \\
\hline & $\geq 31$ & $29.11 \pm 13.89$ & $32.13 \pm 15.41$ \\
\hline & $p^{b}$ & $<0.001^{*}$ & $<0.001^{*}$ \\
\hline & Spearman rho & 0.396 & 0.388 \\
\hline \multirow[t]{2}{*}{ FTND scores } & Spearman rhoc & 0.539 & 0.547 \\
\hline & $\mathrm{p}$ & $<0.001^{*}$ & $<0.001^{*}$ \\
\hline \multirow{2}{*}{$\begin{array}{l}\text { Exhaled CO } \\
\text { levels }\end{array}$} & Spearman $r h o^{c}$ & 0.837 & 0.838 \\
\hline & $\mathrm{p}$ & $<0.001^{*}$ & $<0.001^{*}$ \\
\hline \multirow[t]{3}{*}{ Alcohol use } & No & $22.89 \pm 13.06$ & $25.63 \pm 15.03$ \\
\hline & Yes & $26.4 \pm 13.01$ & $29.34 \pm 14.77$ \\
\hline & $\mathrm{p}^{\mathrm{a}}$ & $0.040^{*}$ & $0.048^{*}$ \\
\hline
\end{tabular}

Data presented as Mean+SD. a Mann-Whitney U test. b Kruskal-Wallis test Pearson correlation analysis. c Spearman rho correlation analysis. *Indicates statistical significance. BMI: body mass index $\left(\mathrm{kg} / \mathrm{m}^{2}\right)$. CO: carbon monoxide. FTND: Fagerström test for nicotine dependence. QTd: QT dispersion. QTcd: corrected OT dispersion.
Table 4 shows the differences in ECG findings concerning addiction levels. No significant relationship was observed between the addiction levels and the longest and the shortest corrected QT values ( $p>0.05$ for both). However, a statistically significant difference was found between addiction levels regarding QTd and QTcd values $(\mathrm{p}<0.001$; $\mathrm{p}<0.001$, respectively).

\section{DISCUSSION}

In the present study, the relationship between smoking and some ECG changes pertaining to increased cardiac arrhythmia risk was investigated, and it was shown that as the level of addiction, the number of cigarettes consumed, exhaled CO levels, and BMI increased, the QT dispersion, which is essential for determining the risk of ventricular arrhythmia, increased.

Smoking is the most crucial, preventable, and modifiable risk factor for cardiovascular system diseases and can cause severe ECG changes that may be associated with ventricular arrhythmia. Especially, $\mathrm{CO}$ and nicotine play an important role in these effects of cigarettes on the cardiovascular system ${ }^{4}$.

In previous studies, the effect of QTd on mortality in the general population was investigated, and it was found that increased QTd raised the risk of cardiac events and mortality ${ }^{21-23}$. The probable prognostic significance of the QTd was evaluated in the preliminary report of the WOSCOPS study. Middleaged men without a history of previous myocardial infarction were examined, and increased future myocardial infarction risk has been demonstrated in those who have QTd $>44 \mathrm{~ms}^{22}$. In another study, healthy volunteers aged $>55$ years were followed up

Table 4. Evaluation of the relationship between FTND addiction levels and ECG parameters $(\mathrm{N}=250)$

\begin{tabular}{lcccc}
\hline $\begin{array}{l}\text { Level of addiction } \\
\text { (FTND score) }\end{array}$ & QTe max & QTe min & QTd \\
\hline Very low (0-2) & $410.15 \pm 32.54$ & $398.31 \pm 35.62$ & $10.85 \pm 8.23$ & $11.85 \pm 8.40$ \\
Low (3-4) & $395.93 \pm 24.27$ & $381.07 \pm 25.12$ & $13.60 \pm 6.77$ & $14.87 \pm 7.22$ \\
Moderate (5) & $402.52 \pm 34.67$ & $378.64 \pm 33.47$ & $21.68 \pm 9.98$ & $23.88 \pm 11.26$ \\
High (6-7) & $405.85 \pm 25.58$ & $381.62 \pm 25.38$ & $21.85 \pm 10.87$ & $24.23 \pm 12.13$ \\
Very high (8-10) & $410.99 \pm 25.35$ & $376.56 \pm 23.95$ & $30.50 \pm 13.56$ & $34.43 \pm 15.78$ \\
p & 0.081 & 0.079 & $<0.001^{*}$ & $<0.001^{*}$
\end{tabular}

Data presented as Mean \pm SD. *p indicates statistical significance. QTc max: corrected QT maximum. QTc min: corrected QT minimum. QTd: OT dispersion. QTcd: corrected QT dispersion. 
for four years, and those with QTcd of $\geq 60 \mathrm{~ms}$ were found to have an increased risk of total mortality, cardiac death, and sudden death ${ }^{21}$. In the study made by Malik et al. ${ }^{23}$ in 2000 , which evaluated $>40$ studies conducted in the last three years related to QTd, the mean QTd values were $33.4 \mathrm{~ms}$ in healthy individuals, $59.2 \mathrm{~ms}$ in patients with previous myocardial infarction, $70.9 \mathrm{~ms}$ in patients with acute myocardial infarction, and $83.2 \mathrm{~ms}$ in patients with long QT syndrome ${ }^{23}$.

In our study, evaluating only chronic smokers, the QTd values ranged 4-64 ms. Although the mean QTd value of smokers was observed to be within normal limits, as the level of addiction, cigarette consumption, and exhaled CO level increased, an increase in QTd was observed, proving the increased risk of arrhythmia with smoking. The highest QTd value we detected in the participants was $64 \mathrm{~ms}$, and this value was higher than for patients who previously had a myocardial infarction.

In the literature, there are studies evaluating the effects of acute and chronic smoking on QT interval and QTd, but different results have been reported from these studies ${ }^{16,24-28}$. Ileri et al. ${ }^{25}$ compared habitual smokers and non-smokers in their study, and chronic cigarette smoking was found to prolong QT interval in healthy people, and habitual smokers had significantly increased QTcd compared to nonsmokers. Although habitual smoking was assessed, the subjects were also assessed under the effect of some acute smoking, because according to the study protocol, they had to smoke 5 cigarettes before the QT measurements were obtained ${ }^{25}$.

In a study investigating the effect of acute smoking on QTd, it was observed that QTd and QTcd increased significantly even after a single cigarette in both healthy smokers and non-smokers, which means that even in chronic smokers, there is no cardiac adaptation and tolerance to the negative effects of smoking, regardless of smoking status; therefore, even one cigarette is thought to increase the risk of arrhythmia $^{16}$.

In the study conducted by Taşolar et al..$^{26}$ comparing healthy chronic smokers and non-smokers, the mean QTd in smokers was $34.2 \pm 8.4 \mathrm{~ms}$, and the mean QTcd was calculated as $37.3 \pm 8.9 \mathrm{~ms}$. In addition to the fact that these parameters were higher in smokers than non-smokers, it was determined that the QTd increased as the degree of smoking increased ${ }^{26}$. In our study, QTd and QTcd values were found approximately $10 \mathrm{~ms}$ longer than in that study.

In addition to the findings of these studies, there was a significant difference between addiction levels in terms of QTd and QTcd values in our study. We found that QT dispersion increased with the increased level of addiction.

In contrast to these studies, Dilaveris et al. ${ }^{27}$ found that only corrected QT interval was prolonged in smokers; however, the QTd did not differ between smokers and non-smokers ${ }^{27}$. Similarly, Karakaya et al. ${ }^{28}$ reported that although a single-dose smokinginduced sympathetic activity, it did not affect QTd.

To our knowledge, even though there are studies in the literature evaluating the relationship between smoking status and QTd and QTcd parameters, the number of studies making a comparison with the level of nicotine addiction is limited. More extensive studies are needed in this area.

Besides the effects of cigarette smoking, in a study evaluating the ECG parameters before and after hookah smoking, the average QTd and QTcd values of the participants measured after the hookah smoking increased statistically significantly from the values obtained before the hookah smoking ${ }^{14}$. Although the average QTd and QTcd values we measured in our study were found to be lower than the values obtained in that study, it is thought that the differences may be due to measurement methods; it is possible that the acute effects of hookah are more significant than those of cigarettes.

CO level, the indicator of cigarette exposure, is related to increased QT dispersion. In previous studies, it has been reported that QT interval and QT dispersion increase in patients with acute CO intoxication ${ }^{29,30}$. Atesçelik et al. ${ }^{29}$ found that both QTd and corrected QTd were longer in CO intoxicated patients than the control group and decreased one week later after intoxication.

We found a significant relationship between the value of $\mathrm{CO}$ measured from expiratory air and QT dispersion and concluded that although it was not high enough to cause intoxication, regardless of the measurement technique, $\mathrm{CO}$ increased the risk of ventricular arrhythmia. However, exhaled CO measurements have some disadvantages. Since the half-life of $\mathrm{CO}$ is short, it is more significant in 
terms of recent exposure. Also, the measured level may be affected due to environmental tobacco exposure and/or non-cigarette $\mathrm{CO}$ sources. Moreover, the QT dispersion may vary for 24 hours. The electrocardiograms of the participants also vary depending on the time of outpatient clinic applications.

\section{CONCLUSIONS}

This study found a significant relationship between the level of addiction, amount of smoking and the exhaled CO levels, and QT dispersion. Our study showed that the risk of arrhythmia increased as the level of addiction increased in smokers. Evaluations in terms of QTd with ECG, are simple and applicable to all smokers and vital in determining the risk of arrhythmia.

\section{REFERENCES}

1. Cohrs S, Rodenbeck A, Riemann D, et al. Impaired sleep quality and sleep duration in smokers-results from the German Multicenter Study on Nicotine Dependence. Addict Biol. 2014;19(3):486-496. doi:10.1111/j.1369-1600.2012.00487.x

2. Aune D, Schlesinger S, Norat T, Riboli E. Tobacco smoking and the risk of sudden cardiac death: a systematic review and meta-analysis of prospective studies. Eur J Epidemiol. 2018;33(6):509-521. doi:10.1007/s10654-017-0351-y

3. Talhout R, Schulz T, Florek E, van Benthem J, Wester P, Opperhuizen A. Hazardous compounds in tobacco smoke. Int J Environ Res Public Health. 2011;8(2):613-628. doi:10.3390/ijerph8020613

4. Wang H, Shi H, Zhang L, et al. Nicotine is a potent blocker of the cardiac A-type $\mathrm{K}(+)$ channels. Effects on cloned Kv4.3 channels and native transient outward current. Circulation. 2000;102(10):1165-1171. doi:10.1161/01.CIR.102.10.1165

5. Moheimani RS, Bhetraratana M, Peters KM, et al. Sympathomimetic effects of acute e-cigarette use: Role of nicotine and non-nicotine constituents. J Am Heart Assoc. 2017;6(9):e006579. doi:10.1161/JAHA.117.006579

6. Bazoukis G, Yeung C, Ho RWH, et al. Association of QT dispersion with mortality and arrhythmic events-A meta-analysis of observational studies. J Arrhythm. 2019;36(1):105-115. doi:10.1002/joa3.12253

7. Sezgin AT, Barutcu I, Ozdemir R, et al. Effect of slow coronary flow on electrocardiographic parameters reflecting ventricular heterogeneity. Angiology. 2007;58(3):289-294. doi:10.1177/0003319707302486

8. Findler M, Birger A, Diamant S, Viskin S. Effects of head-up tilt table test on the QT interval. Ann Noninvasive Electrocardiol. 2010;15(3):245-249. doi:10.1111/j.1542-474X.2010.00371.x
9. Van de Loo A, Arendts W, Hohnloser SH. Variability of QT dispersion measurements in the surface electrocardiogram in patients with acute myocardial infarction and in normal subjects. Am J Cardiol. 1994;74(11):1113-1118. doi:10.1016/0002-9149(94)90462-6

10. Antzelevitch C, Shimizu W, Yan GX, Sicouri S. Cellular basis for QT dispersion. J Electrocardiol. 1998;30 Suppl:168-175. doi:10.1016/S0022-0736(98)80070-8

11. Cardoso CR, Sales MA, Papi JA, Salles GF. QTinterval parameters are increased in systemic lupus erythematosus patients. Lupus. 2005;14(10):846-852. doi:10.1191/0961203305lu2225oa

12. Oluk B. Association between nephropathy and QT dispersion in type 2 diabetic patients. J Surg Med. 2019;3(3):206-209. doi:10.28982/josam.465499

13. Bagrul D, Atik F. Association of vitamin D deficiency with ventricular repolarization abnormalities. Kardiol Pol. 2019;77(9):853-858. doi:10.33963/KP.14888

14. Yıldırım F, Çevik Y, Emektar E, Çorbacıoğlu ŞK, Katırcı Y. Evaluating ECG and carboxyhemoglobin changes due to smoking narghile. Inhal Toxicol. 2016;28(12):546-549. doi:10.1080/08958378.2016.1224957

15. Akilli NB, Akinci E, Akilli H, et al. A new marker for myocardial injury in carbon monoxide poisoning: $\mathrm{T}$ peak-T end. Am J Emerg Med. 2013;31(12):1651-1655. doi:10.1016/j.ajem.2013.08.049

16. Akbarzadeh MA, Yazdani S, Ghaidari ME, et al. Acute effects of smoking on QT dispersion in healthy males. ARYA Atheroscler. 2014;10(2):89-93. PMID:25161676

17. Fagerstrom KO, Schneider NG. Measuring nicotine dependence: a review of the Fagerstrom Tolerance Questionnaire. J Behav Med. 1989;12:159-182. doi:10.1007/BF00846549

18. Uysal MA, Kadakal F, Karşidağ C, Bayram NG, Uysal O, Yilmaz V. Fagerstrom test for nicotine dependence: reliability in a Turkish sample and factor analysis. Tuberk Toraks. 2004;52(2):115-121. PMID:15241694

19. Erb P, Raiff BR, Meredith SE, Dallery J. The accuracy of a lower-cost breath carbon monoxide meter in distinguishing smokers from non-smokers. J Smok Cessat. 2015;10:59-64. doi:10.1017/jsc.2013.37

20. Kallergis EM, Goudis CA, Simantirakis EN, Kochiadakis GE, Vardas PE. Mechanisms, risk factors, and management of acquired long QT syndrome: a comprehensive review. Scientific World Journal. 2012;2012:212178. doi:10.1100/2012/212178

21. de Bruyne MC, Hoes AW, Kors JA, Hofman A, van Bemmel JH, Grobbee DE. QTc dispersion predicts cardiac mortality in the elderly: the Rotterdam Study. Circulation. 1998;97(5):467-472. doi:10.1161/01.CIR.97.5.467

22. Macfarlane PW, Norrie J, WOSCOPS Executive Committee. Looking for prognostic information in the ST-T segment--is it really worth it? J Electrocardiol. 2004;37 Suppl:209-213. doi:10.1016/j.jelectrocard.2004.08.059

23. Malik M, Batchvarov VN. Measurement, interpretation 
and clinical potential of QT dispersion. J Am Coll Cardiol. 2000;36:1749-1766. doi:10.1016/S0735-1097(00)00962-1

24. Singh K. Effect of smoking on QT interval, QT dispersion and rate pressure product. Indian Heart J. 2004;56(2):140-142. PMID:15377136

25. Mleri M, Yetkin E, Tandoğan I, et al. Effect of habitual smoking on QT interval duration and dispersion. Am J Cardiol. 2001;88(3):322-325. doi:10.1016/S0002-9149(01)01653-8

26. Taşolar H, Ballı M, Bayramoğlu A, et al. Effect of smoking on Tp-e interval, Tp-e/QT and Tp-e/QTc ratios as indices of ventricular arrhythmogenesis. Heart Lung Circ. 2014;23(9):827-832. doi:10.1016/j.hlc.2014.03.016

27. Dilaveris P, Pantazis A, Gialafos E, Triposkiadis F, Gialafos J. The Effect of cigarette smoking on the heterogeneity of ventricular repolarization. Am Heart J. 2001;142:833837. doi:10.1067/mhj.2001.118737

28. Karakaya O, Sağlam M, Esen AM, et al. Acute effect of cigarette smoking on ventricular repolarization paramaters. Koşuyolu Heart Journal. 2005;9(1):1-7. https://dergipark. org.tr/en/pub/kkd/issue/10182/125146. Accessed September 21, 2020.

29. Atescelik M, Bozdemir MN, Yildiz M, et al. QT dispersion in carbon monoxide poisoning. Eur Rev Med Pharmacol Sci. 2012;16 Suppl 1:25-29. PMID:22582480

30. Eroglu M, Uz O, Isilak Z, Yalcin M, Yildirim AO, Kardesoglu E. Carbon monoxide poisoning increases Tpeak-Tend dispersion and QTc dispersion. Cardiovasc J Afr. 2014;25(3):106-109. doi:10.5830/CVJA-2014-012
CONFLICTS OF INTEREST

The authors have completed and submitted the ICMJE Form for Disclosure of Potential Conflicts of Interest and none was reported.

FUNDING

There was no source of funding for this research.

AUTHORS' CONTRIBUTIONS

GK: study conception, study design, data collection, data analysis, interpretation of data, revision of manuscript and approval of final version. STK: study conception, study design, data collection, data analysis, interpretation of data, preparation of the first draft, revision of manuscript and approval of final version.

OB: study conception, study design, data analysis, interpretation of data, revision of manuscript and approval of final version.

PROVENANCE AND PEER REVIEW

Not commissioned; externally peer reviewed. 